\title{
Effect of Fucoidan Dietary Supplement on the Chemotherapy Treatment of Patients with Unresectable Advanced Gastric Cancer
}

\author{
Masahide Ikeguchi ${ }^{*}$, Hiroaki Saito², Yasunari Miki³ ${ }^{3}$ Takayuki Kimura3 \\ ${ }^{1}$ Tottori Prefectural Central Hospital, Tottori, Japan \\ ${ }^{2}$ Division of Surgical Oncology, Department of Surgery, Faculty of Medicine, Tottori University, Yonago, Japan \\ ${ }^{3}$ Marine Products Kimuraya, Co., Ltd., Sakaiminato, Japan \\ Email: ${ }^{\text {ikeguchim@pref.tottori.jp }}$
}

Received 5 October 2015; accepted 27 October 2015; published 30 October 2015

Copyright (C) 2015 by authors and Scientific Research Publishing Inc.

This work is licensed under the Creative Commons Attribution International License (CC BY). http://creativecommons.org/licenses/by/4.0/

(c) (i) Open Access

\begin{abstract}
In Japan, S-1 plus cisplatin has become a standard regimen for the treatment of unresectable advanced gastric cancer; however, many patients are unable to continue effective chemotherapy because of the regimen's severe side effects. Thus, control of drug toxicity is key to prolonging patient survival. Fucoidan is a major sulfated polysaccharide found in brown seaweeds and has a wide range of biological activities. In the present study, we analyzed the effect of fucoidan on suppressing the toxicity of chemotherapy drugs. Twenty-four patients with unresectable advanced gastric cancer underwent treatment with $S-1$ plus cisplatin and were randomly allocated into a fucoidan treatment group $(n=12)$ or a control group without fucoidan treatment $(n=12)$. The study results demonstrated that fucoidan controlled the occurrence of fatigue during chemotherapy and patients could continue chemotherapy for longer time periods by maintaining the patients' favorable nourishment status. As a result, the survival of patients with fucoidan treatment was longer than that of patients without fucoidan. Thus, fucoidan should be included as a key food supplement for patients with gastrointestinal carcinomas who are suffering from the adverse side effects of chemotherapy.
\end{abstract}

\section{Keywords}

Chemotherapy, Fucoidan, Gastric Cancer, Toxicity, Prognosis

${ }^{*}$ Corresponding author.

How to cite this paper: Ikeguchi, M., Saito, H., Miki, Y. and Kimura, T. (2015) Effect of Fucoidan Dietary Supplement on the Chemotherapy Treatment of Patients with Unresectable Advanced Gastric Cancer. Journal of Cancer Therapy, 6, 1020-1026. http://dx.doi.org/10.4236/jct.2015.611111 


\section{Introduction}

Outcomes are extremely poor in patients with unresectable gastric cancer. To prolong the survival of these patients, it is important to continue effective chemotherapy for as long as possible. Casaretto et al. [1] reported that chemotherapy increased the 1-year survival rate, provided a longer symptom-free period and improved the quality of life (QOL) of patients. Recently, S-1 plus cisplatin versus S-1 alone for first-line treatment of advanced gastric cancer (SPIRITS) trial identified the S-1 plus cisplatin regimen, which comprises S-1 at a dose of 40 - 60 $\mathrm{mg} / \mathrm{m}^{2}$, in a 5-week cycle (3 weeks on and 2 weeks off), and cisplatin at a dose of $60 \mathrm{mg} / \mathrm{m}^{2}$ cisplatin on day 8 , as a standard first-line treatment for unresectable gastric cancer in Japan [2]. Unfortunately, the severe side effects of this treatment mean it is difficult for most patients to continue the S-1 plus cisplatin regimen [3], and many patients suffer from reduced QOL [4]. Thus, it is clinically important to reduce the toxicity of the chemotherapy regimen so it can be more effective for these patients.

Previously, we reported that fucoidan could reduce the toxicity of combination chemotherapy with oxaliplatin plus 5-fluorouracil (5-FU)/leucovorin (LV) (FOLFOX) or irinotecan plus 5-FU/LV (FOLFIRI) for patients with advanced colorectal cancer [5]. Fucoidan is a sulfated polysaccharide that is found in various species of brown seaweed, such as kombu, wakame, mozuku, and hijiki. It has been reported to have anti-inflammatory, antiviral, and antitumor activity [6]-[8]. Recently, substantial pharmaceutical research has been carried out on fucoidan.

In the present study, we investigated whether fucoidan could reduce the toxicity associated with the chemotherapy treatment of patients who were diagnosed with unresectable gastric cancer at Tottori University Hospital using a randomized study and Eastern Cooperative Oncology Group Performance Status (ECOG PS) 0 and 1.

\section{Methods}

\subsection{Patients}

Between April 2010 and June 2014, 24 patients who were diagnosed with unresectable advanced gastric cancer and who were to undergo S-1 plus cisplatin chemotherapy in our hospital were enrolled in the study. Gastric adenocarcinoma was confirmed histologically by endoscopic biopsy in all patients. The clinical stage of the patients was Stage IV and ECOG PS of these patients was 0 or 1 . They had adequate bone marrow (platelet count $>$ $100,000 / \mathrm{L}$, white blood cell count $>4000 / \mathrm{L}$, granulocyte count $>1500 / \mathrm{L}$, hemoglobin level $>10.0 \mathrm{mg} / \mathrm{dL}$ ), and renal (serum creatinine concentration $<2.0 \mathrm{mg} / \mathrm{dL}$ ) and hepatic (serum bilirubin level $<2.0 \mathrm{mg} / \mathrm{dL}$ ) function. The ethics committee of Tottori University approved this study in 2010 (approval number: 1445). Informed consent was obtained from the 24 patients, and they were randomly allocated into a fucoidan treatment group (n $=12)$ and a control group without fucoidan treatment $(n=12)$. The patients were followed until July 2015. The patient details are shown in Table 1.

\subsection{Chemotherapy}

Standard S-1 plus cisplatin regimen [S-1: $40-60 \mathrm{mg} / \mathrm{m}^{2}$, in a 5-week cycle (3 weeks on and 2 weeks off), in combination with $60 \mathrm{mg} / \mathrm{m}^{2}$ cisplatin on day 8] was administered to all 24 patients [2].

\subsection{Fucoidan Treatment}

Fucoidan is a sulfated polysaccharide that is extracted from brown seaweed. In the present study, we used a high-molecular-weight product of fucoidan derived from Cladosiphon okamuranus (Okinawa mozuku) by Marine Products Kimuraya Co., Ltd. (Tottori, Japan). In the fucoidan group, every patient took $150 \mathrm{~mL} / \mathrm{day}$ of liquid that contained $4.05 \mathrm{~g}$ fucoidan for 6 months after commencing chemotherapy [5].

\subsection{Clinical Assessment}

All toxicities were graded according to the National Cancer Institute Common Toxicity Criteria (NCI CTC) [9]. Hematological variables and clinical status were recorded every 2 weeks during the chemotherapy period. The drug dose levels were reduced in the case of severe or persistent toxicity, according to the protocol [2]. In the case of persistent grade 3 toxicity, chemotherapy was stopped. The occurrence of grade 2 adverse events was compared between both groups. 
Table 1. Patient characteristics.

\begin{tabular}{lccc}
\hline & Fucoidan group & Control & P score \\
\hline Number of patients & 12 & 12 & \\
Age (Mean \pm SD) & $61.2 \pm 11$ & $63.3 \pm 16.2$ & 0.326 \\
Male/female & $7 / 5$ & $6 / 6$ & 0.682 \\
ECOG & $7 / 5$ & $8 / 4$ & 0.673 \\
$\quad$ PS 0/1 & 5 & & \\
Unresectable factors & 3 & 8 & \\
$\quad$ Peritoneal dissemination & 3 & 2 & 0.238 \\
$\quad$ Liver metastasis & 1 & 0 & \\
$\quad$ Distant lymph node metastasis & & 2 & \\
$\quad$ Local advancement & $7 / 5$ & $5 / 7$ & 0.414 \\
\hline Surgical intervention during chemotherapy & & & \\
$\quad$ Yes/no &
\end{tabular}

SD: standard deviation.

Blood samples were taken from each patient routinely before chemotherapy and every 2 weeks during chemotherapy. The serum albumin (ALB) concentration and total lymphocyte count were recorded. The patients' prognostic nutritional indices (PNIs) at 3 months after commencing chemotherapy were compared with their PNIs before chemotherapy as nutritional and immunological parameters of the patients. Each PNI was calculated using the following formula: $10 \times$ serum ALB concentration $(\mathrm{g} / \mathrm{dL})+0.005 \times$ lymphocyte count (number $/ \mathrm{mm}^{3}$ ) in peripheral blood [10].

\subsection{Endpoints}

The patients' survival, measured from the date of commencement of chemotherapy until death, was assessed as the primary endpoint. The incidence and severity of adverse events were also assessed as the secondary endpoints. The continuance of chemotherapy was compared in both groups.

\subsection{Statistical Analysis}

The $\chi$-square test for independence, Fisher's exact probability test, and the Mann-Whitney U test were used to compare patient characteristics, treatment status, adverse events, and antitumor effect. The survival rates of the two groups were estimated by the Kaplan-Meier method, and the statistical differences between survival curves were examined by the log-rank test. A $P$ score of $<0.05$ was regarded as statistically significant.

\section{Results}

All patients were followed up at our hospital. The median follow-up period of the 24 patients was 10 months (range: 3 - 17 months). During the follow-up period, 23 patients (11 in the fucoidan group and 12 in the control group) died because of gastric cancer progression; however, no patient died while undergoing treatment. Additionally, we found no side effects of fucoidan, such as allergic dermatitis. All patients were able to complete fucoidan therapy safely.

The mean survival time (MST) of the 24 patients was 10 months. During the treatment period, 12 of the 24 patients underwent surgical interventions (gastric resection: six and bypass operation: six) because of bleeding from the stomach $(n=6)$ and stenosis $(n=6)$. The MST of the 12 patients with surgical intervention (10 months) was similar to that of the 12 patients without surgical intervention (10 months, $P=0.63)$. In contrast, the MST of the 12 patients who underwent fucoidan treatment (12 months) was significantly better than that of the 12 pa- 
tients without fucoidan treatment (8 months, $P=0.039$, Figure 1).

The observed toxicities ( $\geq$ grade 2 ) of the chemotherapy drugs are listed in Table 2 . We found no patients with severe toxicity (grade 4) in either group. The occurrence of myelosuppression was similar in both the fucoidan and control groups. Appetite loss, nausea and stomatitis caused by S-1 plus cisplatin chemotherapy were not suppressed by fucoidan. In contrast, the occurrence of diarrhea and general fatigue was observed in about $60 \%$ of control group patients, but such adverse events were suppressed in the fucoidan group (the statistical differences were not observed). Because fucoidan was able to suppress diarrhea and fatigue, we found that chemotherapy treatment for these patients could be prolonged. The mean chemotherapy treatment period of the fucoidan group (7.4 months, range: 2 - 12 months) was significantly longer than that of the control group (4.6 months, range: 1 - 10 months, $P=0.044$, Figure 2).

The PNIs of all 24 patients at 3 months after commencing chemotherapy (post-treatment) were compared with those of the patients before treatment (pre-treatment). The PNIs of post-treatment (mean: 43.5, range: 22.1 - 60.7) were similar to those pre-treatment (mean: 44.1, range: 30 - 55.3). The results also indicate that the PNI increased at post-treatment compared with pre-treatment for 14 patients (eight: fucoidan group and six: control group). The MST of the 14 patients with increased PNI at post-treatment (11 months) was significantly better than that of 10 patients with decreased PNI post-treatment (6 months, $P=0.006$ ). Correlation between fucoidan treatment, chemotherapy, and PNI is indicated in Table 3. These results indicate that fucoidan may prevent the deterioration of patients' PNI due to chemotherapy.

Table 2. Major adverse events ( $\geq$ grade 2 ) from the chemotherapy drugs.

\begin{tabular}{cccc}
\hline & Fucoidan group & Control & $P$ \\
\hline Number of patients & 12 & 12 & \\
Leukocytopenia & 0 & 1 & 0.307 \\
Thrombocytopenia & 0 & 1 & 0.307 \\
Appetite loss & 4 & 5 & 0.673 \\
Nausea & 4 & 6 & 0.408 \\
Diarrhea & 0 & 3 & 0.064 \\
Stomatitis & 2 & 2 & - \\
Fatigue & 3 & 7 & 0.098 \\
\hline
\end{tabular}

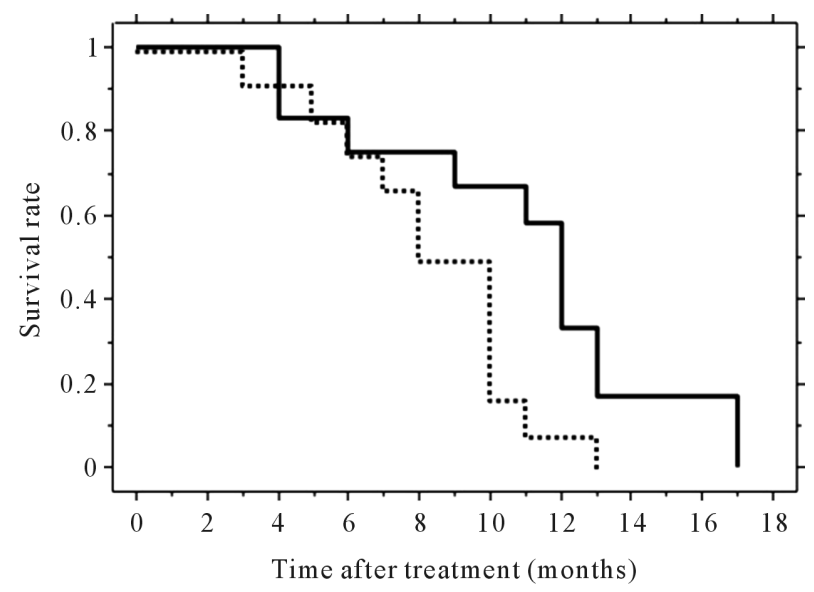

Figure 1. Survival curves of patients with unresectable gastric cancer. Solid line: survival curve of 12 patients with fucoidan treatment. Dotted line: survival curve of 12 patients without fucoidan treatment. The difference was significant $(P$ $=0.039$ ). 
Table 3. Prognostic nutritional indexes (PNIs) before S-1 plus cisplatin treatment (pre-treatment) and at 3 months after commencing chemotherapy (post-treatment).

\begin{tabular}{cccc}
\hline & Pre-treatment & Post-treatment & $P$ \\
\hline Fucoidan + & $45.2 \pm 5.5$ & $47.6 \pm 6.1$ & 0.644 \\
Control & $43 \pm 6.2$ & $39.4 \pm 8.2$ & 0.272 \\
$P$ & 0.273 & 0.028 & \\
\hline
\end{tabular}

Mean \pm SD, SD: standard deviation.

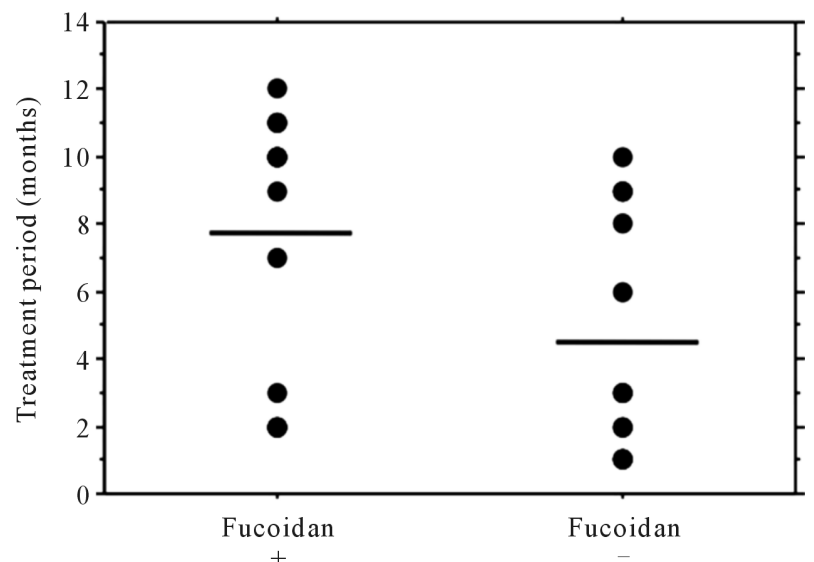

Figure 2. The chemotherapy treatment periods of 12 patients in the fucoidan-treated group were significantly longer than those of the 12 patients in the control group $(P=0.004)$.

\section{Discussion}

Fucoidan is one of the major sulfated polysaccharides of brown seaweeds, such as kombu, wakame, mozuku and hijiki. Recently, substantial research has been carried out on the medical benefits of fucoidan and a wide range of biological activities has been reported. Choi et al. [11] indicated that fucoidan protects the gastric mucosa from inflammatory cytokine-mediated oxidative damage in rats. Hayashi et al. [6] reported that fucoidan reduces CCl4-induced acute and chronic liver failure with hepatic fibrosis and anti-inflammatory activity of fucoidan has been demonstrated in rats [12]. Moreover, it has been demonstrated that fucoidan is not toxic in rats at high doses [13]. Thus, fucoidan has demonstrated potential to improve human health as a supplement rather than as a drug; however, a detailed mechanism of action of fucoidan has not been verified, and its effects in humans have not been reported.

Since the SPIRITS trial, the S-1 plus cisplatin regimen has been recommended in Japan as a standard first-line treatment for unresectable gastric cancer; however, the MST of such patients remains after 10 months [14]. Prolonging the survival of such patients, and continuing the administration of effective chemotherapy as long as possible, are of critical importance. The S-1 plus cisplatin regimen has severe toxicities, such as nausea, vomiting, stomatitis, diarrhea, fatigue, leukocytopenia, and thrombocytopenia. Thus, many patients who suffer from these adverse events may discontinue treatment. Therefore, the prognosis of patients with unresectable gastric cancer is still low even with advances in chemotherapy drug design. To reduce the toxicity of the chemotherapy agents, various types of drugs or dietary supplements have been introduced [15]-[17].

Previously, we found that fucoidan could reduce the toxicities of chemotherapy drugs in patients with colorectal cancer [3]. Thus, in the present study, we analyzed whether fucoidan could protect patients from the toxicities of S-1 plus cisplatin in patients with unresectable gastric cancer and whether this resulted in prolonging the patients' survival. The results indicate that fucoidan suppresses the occurrence of diarrhea and general fatigue during chemotherapy. It has been demonstrated that fatigue graded using NCI CTC was observed in almost 30\% of the treated patients. This fatigue influences the patients' nutritional status, increases morbidity, and can negatively affect the dose intensity of chemotherapy [18]. The addition of fucoidan to the patient reduced the rate of 
grade 2 and 3 fatigue from $60 \%$ to $25 \%$. The addition of fucoidan to the patients' diets also meant that chemotherapy could be continued for longer time periods, and as a result, convalescence of patients tended to be improved. Moreover, fucoidan was able to maintain the nutritional condition of patients during chemotherapy. How fucoidan is able to control general fatigue induced by chemotherapy is unknown, but we will explore this in the future.

Recent research reported that fucoidan is also able to suppress in vitro tumor progression. Yamasaki-Miyamoto et al. [7] and Hyun et al. [19] have demonstrated that fucoidan activates caspase-8 or extracellular signalregulated kinase and induced apoptosis in tumor cells. The pro-apoptotic effects of fucoidan have been shown in a gastric cancer cell line [20]. In the present study, even though the number of patients was small, the prognosis of patients with unresectable advanced gastric cancer was better with fucoidan treatment compared with those patients not supplemented with fucoidan. One reason could be that fucoidan prolonged chemotherapy duration by suppressing the toxicity of the chemotherapy drugs. Another reason might be an anticancer effect of fucoidan itself. This highlights the need for larger controlled studies to evaluate the therapeutic effect of fucoidan for patients with gastrointestinal carcinoma.

\section{Competing Interests}

We have no competing interests about this manuscript.

\section{References}

[1] Casaretto, L., Sousa, P.L.R. and Mari, J.J. (2006) Chemotherapy versus Support Cancer Treatment in Advanced Gastric Cancer: A Meta-Analysis. Brazilian Journal of Medical and Biological Research, 39, 431-440. http://dx.doi.org/10.1590/S0100-879X2006000400002

[2] Koizumi, W., Narahara, H., Hara, T., Takagane, A., Akiya, T., Takagi, M., Miyashita, K., Nishizaki, T., Kobayashi, O., Takiyama, W., Toh, Y., Nagaie, T., Takagi, S., Yamamura, Y., Yanaoka, K., Orita, H. and Takeuchi, M. (2008) S-1 plus Cisplatin versus S-1 Alone for First-Line Treatment of Advanced Gastric Cancer (SPIRITS trial): A Phase III Trial. The Lancet Oncology, 9, 215-221. http://dx.doi.org/10.1016/S1470-2045(08)70035-4

[3] Ikeguchi, M., Kader, A., Yoshimoto, M., Takaya, S., Watanabe, J., Fukumoto, Y., Osaki, T., Saito, H., Tatebe, S. and Wakatsuki, T. (2013) Usefulness of Palliative Prognostic Score in the Treatment of Patients with Non-Resectable Gastric Cancer. Molecular and Clinical Oncology, 1, 253-256. http://dx.doi.org/10.3892/mco.2013.66

[4] Koo, D.H., Ryu, M.H., Ryoo, B.Y., Lee, S.S., Moon, J.H., Chang, H.M., Lee, J.L., Kim, T.W. and Kang, Y.K. (2012) Three-Week Combination Chemotherapy with S-1 and Cisplatin as First-Line Treatment in Patients with Advanced Gastric Cancer: A Retrospective Study with 159 Patients. Gastric Cancer, 15, 305-312. http://dx.doi.org/10.1007/s10120-011-0117-2

[5] Ikeguchi, M., Yamamoto, M., Arai, Y., Maeta, Y., Ashida, K., Katano, K., Miki, Y. and Kimura, T. (2011) Fucoidan Reduces the Toxicities of Chemotherapy for Patients with Unresectable Advanced or Recurrent Colorectal Cancer. Oncology Letters, 2, 319-322. http://dx.doi.org/10.3892/ol.2011.254

[6] Hayashi, S., Itoh, A., Isoda, K., Kondoh, M., Kawase, M. and Yagi, K. (2008) Fucoidan Partly Prevents CCl4-Induced Liver Fibrosis. European Journal of Pharmacology, 580, 380-384. http://dx.doi.org/10.1016/j.ejphar.2007.11.015

[7] Yamasaki-Miyamoto, Y., Yamasaki, M., Tachibana, H. and Yamada, K. (2009) Fucoidan Induces Apoptosis through Activation of Caspase-8 on Human Breast Cancer MCF-7 Cells. Journal of Agricultural and Food Chemistry, 57, 8677-8682. http://dx.doi.org/10.1021/jf9010406

[8] Saitoh, Y., Nagai, Y. and Miwa, N. (2009) Fucoidan-Vitamin C Complex Suppresses Tumor Invasion through the Basement Membrane, with Scarce Injuries to Normal or Tumor Cells, via Decreases in Oxidative Stress and Matrix Metalloproteinases. International Journal of Oncology, 35, 1183-1189.

[9] Therasse, P., Arbuck, S.G., Eisenhauer, E., Wanders, J., Kaplan, R.S., Rubinstein, L., Verweij, J., van Glabbeke, M., van Oosterom, A.T., Christian, M.C. and Gwyther, S.G. (2000) New Guidelines to Evaluate the Response to Treatment in Solid Tumors. Journal of the National Cancer Institute, 92, 205-216. http://dx.doi.org/10.1093/jnci/92.3.205

[10] Nozoe, T., Kohno, M., Iguchi, T., Mori, E., Maeda, T., Matsukuma, A. and Ezaki, T. (2012) The Prognostic Nutritional Index Can Be a Prognostic Indicator in Colorectal Carcinoma. Surgery Today, 42, 532-535. http://dx.doi.org/10.1007/s00595-011-0061-0

[11] Choi, J.I., Raghavendran, H.R.B., Sung, N.Y., Kim, J.H., Chun, B.S., Ahn, D.H., Choi, H.S., Kang, K.W. and Lee, J.W. (2010) Effect of Fucoidan on Aspirin-Induced Stomach Ulceration in Rats. Chemico-Biological Interactions, 183, 249-254. http://dx.doi.org/10.1016/j.cbi.2009.09.015 
[12] Del Bigio, M.R., Yan, H.J., Campbell, T.M. and Peeling, J. (1999) Effect of Fucoidan Treatment on CollagenaseInduced Intracerebral Hemorrhage in Rats. Journal of Neurology Research, 21, 415-419.

[13] Kim, K.J., Lee, O.H., Lee, H.H. and Lee, B.Y. (2010) A 4-Week Repeated Oral Dose Toxicity Study of Fucoidan from the Sporophyll of Undaria pinnatifida in Sprague-Dawley Rats. Toxicology, 267, 154-158. http://dx.doi.org/10.1016/j.tox.2009.11.007

[14] Shinoda, M., Ando, T., El-Omar, E.M., Takashi, H., Suzuki, T., Murayama, M., Morise, K. and Goto, H. (2012) Programmed Chemotherapy for Patients with Metastatic Unresectable Gastric Cancer. PLoS One, 7, e38652. http://dx.doi.org/10.1371/journal.pone.0038652

[15] Lee, C.K., Park, K.K., Hwang, J.K., Lee, S.K. and Chung, W.Y. (2009) Extract of Prunus persica Flesh (PPFE) Improves Chemotherapeutic Efficacy and Protects against Nephrotoxicity in Cisplatin-Treated Mice. Phytotherapy Research, 23, 999-1005. http://dx.doi.org/10.1002/ptr.2740

[16] Block, K.I., Koch, A.C., Mead, M.N., Tothy, P.K., Newman, R.A and Gyllenhaal, C. (2008) Impact of Antioxidant Supplementation on Chemotherapeutic Toxicity: A Systematic Review of the Evidence from Randomized Controlled Trials. International Journal of Cancer, 123, 1227-1239. http://dx.doi.org/10.1002/ijc.23754

[17] Eussen, S., Klungel, O., Garssen, J., Verhagen, H., van Kranen, H., van Loveren, H. and Rompelberg, C. (2010) Support of Drug Therapy Using Functional Foods and Dietary Supplements: Focus on Statin Therapy. British Journal of Nutrition, 103, 1260-1277. http://dx.doi.org/10.1017/S0007114509993230

[18] Iop, A., Manfredi, A.M. and Bonura, S. (2004) Fatigue in Cancer Patients Receiving Chemotherapy: An Analysis of Published Studies. Annals of Oncology, 15, 712-720. http://dx.doi.org/10.1093/annonc/mdh102

[19] Hyun, J.H., Kim, S.C., Kang, J.I., Kim, M.K., Boo, H.J., Kwon, J.M., Koh, Y.S., Hyun, J.W., Park, D.B., Yoo, E.S. and Kang, H.K. (2009) Apoptosis Inducing Activity Of fucoidan in HCT-15 Colon Carcinoma Cells. Biological \& Pharmaceutical Bulletin, 32, 1760-1764. http://dx.doi.org/10.1248/bpb.32.1760

[20] Yoshimoto, M., Higaki, K., Nanba, E. and Ikeguchi, M. (2015) Anti-Proliferation Activity of Fucoidan in MKN45 Gastric Cancer Cells and Downregulation of Phosphorylated ASK1, a Cell Cycle-Regulated Kinase. Yonago Acta Medica, 58, 1-7. 